\title{
HERMITE-HADAMARD TYPE INEQUALITIES FOR HARMONICALLY CONVEX STOCHASTIC PROCESSES
}

\author{
Nurgül OKUR ${ }^{1} \quad$ Imdat işCAN² $\quad$ Emine YÜKSEK DIZDAR
}

\begin{abstract}
In this study are investigated harmonically convex stochastic processes which are an extensions of convex stochastic processes. Suitable examples are also given for these types of processes. In addition, in this case a harmonic convex stochastic process is increasing or decreasing, the relation with convexity is revealed. The concepts of convexity and inequality have an important place in literature, since it provides a broader setting to study the optimization and mathematical programming problems. The obtained results in this study are necessary to compare the maximum and minimum values of of a stochastic process with the expected value of its which has a probability density function, is particularly harmonic convex. Therefore, Hermite-Hadamard type inequalities for harmonically convex stochastic processes and some boundaries for these inequalities are obtained. There are used as methods concepts of mean-square continuity, mean-square differentiability, mean-square integrability and monotonicity for stochastic processes in this study.
\end{abstract}

Key Words: Harmonically convex stochastic processes, mean-square continuity, monotonicity, mean-square differentiablity, mean square integrability, Hermite-Hadamard inequality

JEL Codes: C690, C020, C1

\section{HARMONIK KONVEKS STOKASTIK SÜREÇLER IÇIN HERMITE-HADAMARD TIPLI EŞiTSIZLIKLER}

\section{Öz}

Bu çalışmada, konveks stokastik süreçlerin bir genişlemesi olan harmonik konveks stokastik süreçler matematiksel olarak incelenmiştir. Bu tip süreçler için uygun örnekler de verilmiştir. Ayrıca, bir harmonik konveks stokastik sürecin artan veya azalan olması durumunda, konvekslikle ilişkisi ortaya konulmuştur. Konvekslik ve eşitsizlik kavramları, optimizasyon ve matematiksel programlama problemlerini incelemek için daha geniş bir çalışma imkanı sağladığı için, literatürde önemli bir yere sahiptir. Bu çalışmada bulunun sonuçlar, olasılık yoğunluk fonksiyonu özellikle harmonik konveks olan bir stokastik sürecin beklenen değeri ile maximum ve minimum değerlerinin karşılaştırılmasında gereklidir. Bu nedenle, harmonik konveks stokastik süreçler için Hermite-Hadamard tipli eşitsizlikler ve bu eşitsizlikler için bazı sınırlar elde edilmiştir. Bu çalışmada metot olarak, stokastik süreçler için orta-kuadratik anlamda süreklilik, orta-kuadratik anlamda türevlenebilirlik, orta-kuadratik anlamda integrallenebilirlik ve monotonluk kavramları kullanılmıştır.

Anahtar Kelimeler: Harmonik konveks stokastik süreçler, orta-kuadratik anlamda süreklilik, monotonluk, ortakuadratik anlamda differensiyellenebilirlik, orta-kuadratik anlamda integrallenebilirlik, Hermite-Hadamard eşitsizliği

JEL Kodları: C690, C020, C1

\footnotetext{
${ }^{1}$ Yrd. Doç. Dr., Giresun University, Department of Statistics, Giresun, Turkey, nrgokur@gmail.com

${ }^{2}$ Doç. Dr., Giresun University, Department of Mathematics, Giresun, Turkey, imdati@gmail.com

${ }^{3}$ Giresun AKSA Elektrik A.S.., Giresun, Turkey, eminee_yuksekk@hotmail.com
} 


\section{Introduction}

It is well known that, for every real convex function $f$ on the interval $[a, b]$, we have

$$
f\left(\frac{a+b}{2}\right) \leq \frac{1}{b-a} \int_{a}^{b} f(x) d x \leq \frac{f(a)+f(b)}{2} .
$$

These are celebrated Hermite-Hadamard inequalities. In probabilistic words, they say that

$$
f(E X) \leq_{c x} E f(X) \leq_{c x} E f\left(X^{*}\right), f \in \mathcal{C}_{c x}
$$

where $E$ denotes mathematical expectation, $X$ (respextively, $X^{*}$ ) is a random variable having the uniform distribution on the interval $[a, b]$ (respectively, on the set $\{a, b\}$ ), and $\mathcal{C}_{c x}$ is the set of all real convex functions on $[a, b] ; \leq_{c x}$ stands for the so called convex order of random variables (J. de la Cal et al, (2006)).

Convexity of sets and functions are extremely simple notions to define, so it may be somewhat surprising the depth and breadth of ideas that these notions give rise to. It turns out that convexity is central to a vast number of applied areas, including Statistical Mechanics, Thermodynamics, Mathematical Economics, and Statistics, and that many inequalities, including Hölder's and Minkowski's inequalities, are related to convexity (Simon, (2011)).

More than twenty years after Hermite's work was published, J. L. W. V. Jensen, the celebrated Danish engineer and mathematician, anticipated in 1906:

"It seems to me that the notion of convex function is about as fundamental that these: positive function, increasing function. If I'm not mistaken in this, the notion will have to find its place in the exhibitions of the theory of real functions"

the concept of convex functions has indeed found an important place in Modern Mathematics as can be seen in a large number of research articles and books devoted to the field these days. In this context, the Hermite-Hadamard inequality, which, we can say, is the first fundamental result for convex functions with a natural geometrical interpretation and many applications, has attracted and continues to attract much interest in elementary mathematics.

Many mathematicians have devoted their efforts to generalise, refine, counterpart and extend it for different classes of functions such as: quasi-convex functions, Godunova-Levin class of functions, log-convex and r-convex functions, $p$-functions, etc or apply it for special means (p-logarithmic means, identric mean, Stolarsky means, etc) (Beckenbach, et al,(1961))

Since the publications of the two papers in 1905 and 1906 by J. L. W. V. Jensen, the theory of convex functions has experienced a rapid development. This can be attributed to several causes: first, a great many areas in modern analysis directly or indirectly involve the application of convex functions; secondly, convex functions are closely related to the theory of inequalities, and many important inequalities are consequences of the applications of convex functions. For example, the important AG inequality or the general inequality between means of orders rand s, such as Holder's and Minkowski's inequalities, are all consequences of Jensen's inequality for convex functions. As a result, the topic of convex functions has been treated extensively in the classical book by Hardy, Littlewood, and Polya, Beckenbach, Bellman and Mitrinovic. An earlier book that is devoted solely to inequalities for convex functions was written by Pecaric in 1987 (Josip et al, (1992))

The field of stochastic processes is essentially a branch of probability theory, treating probabilistic models that evolve in time. It is best viewed as a branch of mathematics, starting with the axioms of probability and containing a rich and fascinating set of results following from those axioms. Although the results are applicable to many areas, they are best understood initially in terms of their mathematical structure and interrelationships. Applying axiomatic probability results to a real-world area requires creating a probability model for the given area. Mathematically precise results can then be derived within the model and translated back to the real world. If the model fits the area sufficiently well, real problems can be solved by analysis within 
the model. However, since models are almost always simplified approximations of reality, precise results within the model become approximations in the real world. Choosing an appropriate probability model is an essential part of this process. Sometimes an application area will have customary choices of models, or at least structured ways of selecting them. For example, there is a well-developed taxonomy of queueing models. A sound knowledge of the application area, combined with a sound knowledge of the behavior of these queueing models, often lets one choose a suitable model for a given issue within the application area. In other cases, one can start with a particularly simple model and use the behavior of that model to gain insight about the application, and use this to iteratively guide the selection of more general models. An important aspect of choosing a probability model for a real-world area is that a prospective choice depends heavily on prior understanding, at both an intuitive and mathematical level, of results from the range of mathematical models that might be involved (Galleger, (2013))

There are various ways to define stochastic monotonicity and convexity for stochastic processes, and it is of great importance in optimization, especially in optimal designs, and also useful for numerical approximations when there exist probabilistic quantities in the literature.

In 1980 Nikodem proposed convex stochastic processes and gave some properties which are also known for classical convex functions. Jensen-convex, $\lambda$-convex stochastic processes were introduced by Skowronski in 1992. In 2012 Kotrys extended the classical Hermite-Hadamard inequality to convex stochastic processes. There are many studies in recent years on some types of convexity for stochastic processes and Hermite-Hadamard inequalities for related convex stochastic processes in the literature.

The author's findings led to our motivation to build our work. The main goal is to introduce harmonically convex stochastic processes. Moreover, we prove Hermite-Hadamard type inequalities for harmonically convex stochastic processes and obtain some important results for these processes.

\section{Methods}

There are as methods concepts probability theory, random variables, probability measure, and types of convergence as follows:

Probability theory is a fundamental pillar of modern mathematics with relations to other mathematical areas like algebra, topology, analysis, geometry or dynamical systems. As with any fundamental mathematical construction, the theory starts by adding more structure to a set $\Omega$.

In a similar way as introducing algebraic operations, a topology, or a time evolution on a set, probability theory adds a measure theoretical structure to $\Omega$ which generalizes "counting" on finite sets: in order to measure the probability of a subset $A \subset \Omega$, one singles out a class of subsets $\mathfrak{J}$, on which one can hope to do so. This leads to the notion of a $\sigma$-algebra $\mathfrak{J}$. It is a set of subsets of $\Omega$ in which on can perform finitely or countably many operations like taking unions, complements or intersections. The elements in $A \subset \Omega$ are called events. If a point $\omega$ in the "laboratory" $\Omega$ denotes an "experiment", an "event" $A \in \mathfrak{J}$ is a subset of $\Omega$, for which one can assign a probability $P[A] \in[0,1]$.

The probability measure $P$ has to satisfy obvious properties like that the union $A \cup B$ of two disjoint events $A, B$ satisfies $P[A \cup B]=P[A]+P[B]$ or that the complement $A_{c}$ of an event $A$ has the probability $P\left[A_{c}\right]=1-P[A]$.

If $\Omega$ is a subset of an Euclidean space like the plane, $P[A]=\int_{A} f(x, y) d x d y$ for a suitable nonnegative function $f$, we are led to integration problems in calculus.

Actually, in many applications, the probability space is part of Euclidean space and the $\sigma$-algebra is the smallest which contains all open sets. It is called the Borel $\sigma$-algebra. An important example is the Borel $\sigma$-algebra on the real line. 
Given a probability space $(\Omega, \mathfrak{I}, P)$, one can define random variables X. A random variable is a function $X$ from $\Omega$ to the real line $\mathbb{R}$ which is measurable in the sense that the inverse of a measurable Borel set $B$ in $R$ is in $\mathfrak{I}$. The interpretation is that if $\omega$ is an experiment, then $X(\omega)$ measures an observable quantity of the experiment.

In probability theory, where functions are often denoted with capital letters, like $X, Y, \ldots, a$ random variable $X$ is measurable if $X^{-1}(B) \in \mathfrak{I}$ for all Borel sets $B \in \mathcal{B}$. Any continuous function is measurable for the Borel $\sigma$-algebra. As in calculus, where one does not have to worry about continuity most of the time, also in probability theory, one often does not have to sweat about measurability issues.

Random variables form so an algebra $\mathcal{L}$. The expectation of a random variable $X$ is denoted by $E[X]$ if it exists. It is a real number which indicates the "mean" or "average" of the observation $X$. It is the value, one would expect to measure in the experiment. If $X=1_{B}$ is the random variable which has the value 1 , if $\omega$ is in the event $B$ and 0 , if $\omega$ is not in the event $B$, then the expectation of $X$ is just the probability of $B$.

The constant random variable $X(\omega)=a$ has the expectation $E[X]=a$. These two basic examples as well as the linearity requirement $E[a X+b Y]=a E[X]+b E[Y]$ determine the expectation for all random variables in the algebra $\mathcal{L}$ : first one defines expectation for finite sums $\sum_{i=1}^{n} a_{i} 1_{B_{i}}$ called elementary random variables, which approximate general measurable functions.

Extending the expectation to a subset $\mathcal{L}^{1}$ of the entire algebra is part of integration theory. While in calculus, one can live with the Riemann integral on the real line, which defines the integral by Riemann sums $\int_{a}^{b} f(x) d x \sim \frac{1}{n} \sum_{i / n \in[a, b]} f(i / n)$, the integral defined in measure theory is the Lebesgue integral. The later is more fundamental and probability theory is a major motivator for using it. It allows to make statements like that the probability of the set of real numbers with periodic decimal expansion has probability 0 . In general, the probability of $A$ is the expectation of the random variable $X(x)=f(x)=1_{A(x)}$.

In calculus, the integral $\int_{0}^{1} f(x) d x$ would not be defined because a Riemann integral can give 1 or 0 depending on how the Riemann approximation is done. Probability theory allows to introduce the Lebesgue integral by defining $\int_{a}^{b} f(x) d x$ as the limit of $\frac{1}{n} \sum_{i=1}^{n} f\left(x_{i}\right)$ for $n \rightarrow \infty$, where $x_{i}$ are random uniformly distributed points in the interval $[a, b]$. This Monte Carlo definition of the Lebesgue integral is based on the law of large numbers and is as intuitive to state as the Riemann integral which is the limit of $\frac{1}{n} \sum_{x_{j}=j / n \in[a, b]} f\left(x_{j}\right)$ for $n \rightarrow \infty$.

With the fundamental notion of expectation one can define the variance,

$$
\operatorname{Var}[X]=E\left[X^{2}\right]-E[X]^{2}
$$

and the standard deviation $\sigma[X]=\sqrt{\operatorname{Var}[X]}$ of a random variable $\mathrm{X}$ for which $X^{2} \in \mathcal{L}^{1}$.

$\mathrm{X}$ can be described well by its distribution function $F_{x}$. This is a real-valued function defined as

$$
F_{x}(s)=P[X \leq s]
$$

on $\mathbb{R}$, where $\{X \leq s\}$ is the event of all experiments $\omega$ satisfying $X(\omega) \leq s$.

The distribution function does not encode the internal structure of the random variable $X$; it does not reveal the structure of the probability space for example. But the function $F_{x}$ allows the construction of a probability space with exactly this distribution function. There are two important types of distributions, continuous distributions with a probability density function $f_{x}=F_{x}^{\prime}$ and discrete distributions for which $F$ is piecewise constant.

The law $\mu_{\mathrm{x}}$ of the random variable is a probability measure on the real line satisfying 


$$
\mu_{x}((a, b])=F_{x}(b)-F_{x}(a) \text {. }
$$

By the Lebesgue decomposition theorem, one can decompose any measure $\mu$ into a discrete part $\mu_{p p}$, an absolutely continuous part $\mu_{a c}$ and a singular continuous part $\mu_{s c}$. Random variables $\mathrm{X}$ for which $\mu_{x}$ is a discrete measure are called discrete random variables, random variables with a continuous law are called continuous random variables.

Inequalities play an important role in probability theory. The Chebychev inequality

$$
P[|X-E[X]| \geq c] \leq \frac{\operatorname{Var}[X]}{c^{2}}
$$

is used very often. It is a special case of the Chebychev-Markov inequality

$$
h(c) \cdot P[X \geq c] \leq E[h(X)]
$$

for monotone nonnegative functions $h$. Other inequalities are the Jensen inequality

$$
E[h(X)] \geq h(E[X])
$$

for convex functions $h$, the Minkowski inequality

$$
\|X+Y\|_{p} \leq\|X\|_{p}+\|Y\|_{p}
$$

or the Hölder inequality

$$
\|X Y\|_{1} \leq\|X\|_{p}\|Y\|_{q}, 1 / p+1 / q=1
$$

for random variables, $X, Y$, for which $\|X\|_{p}=E\left[|X|^{p}\right],\|Y\|_{q}=E\left[|Y|^{q}\right]$ are finite. Any inequality which appears in analysis can be useful in the toolbox of probability theory (Knill, (2008)).

Here, we would like to provide definitions of different types of convergence and discuss how they are related. Consider a sequence of random varibles $X_{1}, X_{2}, X_{3}, \ldots$, i.e, $\left\{X_{n}, n \in \mathbb{N}\right\}$. This sequence might "converge" to a random variable $X$. There are four types of convergence such that convergence in distibution, convergence in probability, convergence in mean, and almost sure convergence. These are all different kinds of convergence. A sequence might converge in one sense but not another. Some of these convergence types are "stronger" than others and some are "weaker".

Convergence in distribution is in some sense the weakest type of convergence. All it says is that the cumulative distribution function of $X_{n}$ 's converges to the cumulative distribution function of $X$ as $n$ goes to infinity. It does not require any dependence between the $X_{n}$ 's and $X$. We saw this type of convergence before when we discussed the central limit theorem. To say that $X_{n}$ converges in distribution to $X$, we write $X_{n} \stackrel{d}{\rightarrow} X$.

Convergence in probability is stronger than convergence in distribution. In particular, for a sequence $X_{1}, X_{2}, X_{3}, \ldots$ to converge to a random variable $X$, we must have that $P\left(\left|X_{n}-X\right| \geq \epsilon\right)$ goes to 0 as $n \rightarrow \infty$ for any $\epsilon>0$. To say that $X_{n}$ converges in probability to $X$, we write $X_{n} \stackrel{P}{\rightarrow} X$.

One way of interpreting the convergence of a sequence $X_{n}$ to $X$ is to say that the "distance" between $X$ and $X_{n}$ is getting smaller and smaller. For example, if we define the distance between $X_{n}$ and $X$ as $P\left(\left|X_{n}-X\right| \geq \epsilon\right)$, we have convergence in probability. One way to define the distance between $X_{n}$ and $X$ is $E\left(\left|X_{n}-X\right| r\right)$, where $r \geq 1$ is a fixed number. This refers to convergence in mean. For convergence in mean, it is usually required that $E\left|X_{n}^{r}\right|<\infty$. The most common choice is $r=2$, in which case it is called the mean-square convergence. Some authors refer to the case $r=1$ as convergence in mean.

Consider a sequence of random variables $X_{1}, X_{2}, X_{3}, \ldots$ that is defined on an underlying sample space $S$. For simplicity, let us assume that $S$ is a finite set, so we can write $S=\left\{s_{1}, s_{2}, \cdots, s_{k}\right\}$. Remember that each $X_{n}$ is a function from $S$ to the set of real numbers. Thus, we may write $X_{n}\left(s_{i}\right)=x_{n i}$, for $i=1,2, \ldots, k$. After this random experiment is performed, one of $\mathrm{si}_{i}$ is outcome of the experiment, we 
observe the sequence $x_{1 j}, x_{2 j}, x_{3 j}, \ldots$. Since this is a sequence of real numbers, we can talk about its convergence. Almost sure convergence is defined based on the convergence of such sequences (Suhov, (2005)).

The above mentioned mean-square convergence is used throughout this paper.

There are definitions of concepts such as mean-square continuity, mean-square differentiability, mean-square integrability and monotonicity for stochastic processes as follows.

Let's see mathematical definition of a stochastic process:

Definition 2.1. Let $(\Omega, \mathfrak{J}, P)$ be an arbitrary probability space. A function $X: \Omega \rightarrow \mathbb{R}$ is called a random variable if it is $\mathfrak{I}$-measurable. A stochastic process $X: I \times \Omega \rightarrow \mathbb{R}$, where $I \subset \mathbb{R}$ is an interval, is called a stochastic process if for every $t \in I$ the function $X(t,$.$) is a random variable$ (Kotrys (2012)).

Let's recall some important types of convexity for stochastic processes:

Definition 2.2. Let $(\Omega, \Im, P)$ be an arbitrary probability space and $I \subset \mathbb{R}$ be an interval. We say that a stochastic process $X: I \times \Omega \rightarrow \mathbb{R}$ is

(i) convex if $X(\lambda u+(1-\lambda) v,.) \leq \lambda X(u,)+.(1-\lambda) X(v,$.$) for all u, v \in I$ and $\lambda \in[0,1]$,

(ii) $\lambda$-convex if $X(\lambda u+(1-\lambda) v,.) \leq \lambda X(u,)+.(1-\lambda) X(v,$.$) for all u, v \in I$ and $\lambda$ is a fixed number on $(0,1)$.

(iii) Jensen-convex if

$$
X\left(\frac{u+v}{2}, .\right) \leq \frac{X(u, .)+X(v, .)}{2}
$$

for all $u, v \in I$ (Kotrys (2012)).

Let's give some basic definitions and notions about continuity concepts and differentiability for stochastic processes, mean-square integral of a stochastic process.

Definition 2.3. Let $(\Omega, \mathfrak{I}, P)$ be an arbitrary probability space and $I \subset \mathbb{R}$ be an interval. We say that a stochastic process $X: I \times \Omega \rightarrow \mathbb{R}$ is called

(i) continuous in probability on $I$ if for all $t_{0} \in I$ if

$$
P-\lim _{t \rightarrow t_{0}} X(t, \cdot)=X\left(t_{0}, \cdot\right)
$$

where $P$ - lim denotes limit in probability,

(ii) mean-square continuous on $I$ if for all $t_{0} \in I$ if

$$
\lim _{t \rightarrow t_{0}} E\left[X(t, \cdot)-X\left(t_{0}, \cdot\right)\right]^{2}=0
$$

where $E[X(t, \cdot)]$ denotes expectation value of the random variable $X(t, \cdot)$,

(iii) increasing (decreasing) if for all $u, v \in I$ such that $u<v$ if $X(u, \cdot) \leq X(v, \cdot)(X(u, \cdot) \geq X(v, \cdot))$,

(iv) monotonic if it is increasing or decreasing,

(v) mean-square differentiable at a point if $\mathrm{t} \in \mathrm{I}$ there is random variable $X^{\prime}(t, \cdot): I \times \Omega \rightarrow \mathbb{R}$ such that

$$
X^{\prime}(t)=P-\lim _{t \rightarrow t_{0}} \frac{X(t, \cdot)-X\left(t_{0}, \cdot\right)}{t-t_{0}}
$$


We say that a stochastic process $X: I \times \Omega \rightarrow \mathbb{R}$ is cont inous (differentiable) if it is continuous (differentiable) at every point of interval $I$ (Kotrys (2012)).

Definition 2.4. Let $(\Omega, \mathfrak{J}, P)$ be an arbitrary probability space and $I \subset \mathbb{R}$ be an interval and $X: I \times \Omega \rightarrow \mathbb{R}$ be a stochastic process with $E\left[X\left(t^{2}\right)\right] \leq \infty$ for all $t \in I$. Let $[u, v] \subset I, u=t_{0}<$ $t_{1} \ldots<t_{n}=v$ be a partition of $[u, v]$ and $\Theta_{k} \in\left[t_{k-1}, t_{k}\right]$ arbitrarily fork $=1, \ldots, n$. A random variable $Y: \Omega \rightarrow \mathbb{R}$ is called mean-square integral of the process $\mathrm{X}(\mathrm{t},$.$) on [u, v]$ if the following identity holds (Kotrys (2012)):

$$
\lim _{n \rightarrow \infty} E\left(\sum_{k=1}^{n} X\left(\Theta_{k}\right) \cdot\left(t_{k}-t_{k-1}\right)-Y\right)^{2}=0 .
$$

Then we can write

$$
\int_{u}^{v} X(t, \cdot) d t=Y(.)
$$

Mean-square integral operator is increasing, that is,

$$
\int_{u}^{v} X(t, \cdot) d t \leq \int_{u}^{v} Z(t, \cdot) d t
$$

where $X(t, \cdot) \leq Z(t, \cdot)$ on $[u, v]$.

Now, we give the well-known Hermite-Hadamard integral inequality for convex stochastic processes (Kotrys (2012)):

Theorem 2.1. If $X: I \times \Omega \rightarrow \mathbb{R}$ is Jensen-convex and mean square continuous in the interval $I \times$ $\Omega$, then for any $u, v \in I, u<v$ we have

$$
X\left(\frac{u+v}{2}, \cdot\right) \leq \frac{1}{v-u} \int_{u}^{v} X(t, \cdot) d t \leq \frac{X(u, \cdot)+X(v, \cdot)}{2} .
$$

\section{Findings}

The main subject of this paper is to extend some well-known result concerning harmonically convex functions to harmonically convex stochastic processes. Also, we purpose to obtain HermiteHadamard type inequalities for harmonically convex stochastic processes.

Definition 3.1. Let $I \subset \mathbb{R} \backslash\{0\}$ be a real interval. A stochastic process $X: I \times \Omega \rightarrow \mathbb{R}$ is said to be harmonically convex stochastic process, if

$$
X\left(\frac{u v}{\lambda u+(1-\lambda) v^{\prime}},\right) \leq \lambda X(v, \cdot)+(1-\lambda) X(u, \cdot)
$$

for all $u, v \in I$ and $\lambda \in[0,1]$. If the inequality (1) is reversed, then $X$ is said to be harmonically concave.

Example 3.1. Let $X:(0, \infty) \times \Omega \rightarrow \mathbb{R}, X(u, \cdot)=u$, and $Y:(-\infty, 0) \times \Omega \rightarrow \mathbb{R}, Y(u, \cdot)=u$, then $X$ is a harmonically convex and $Y$ is a harmonically concave stochastic process. then

Proposition 3.1. Let $I \subset \mathbb{R} \backslash\{0\}$ be a real interval and $X: I \times \Omega \rightarrow \mathbb{R}$ be a stochastic process,

- $\quad$ if $I \subset(0, \infty)$ and $X$ is convex and nondecreasing stochastic process then $X$ is harmonically convex,

- if $I \subset(0, \infty)$ and $X$ is harmonically convex and nondecreasing stochastic process then $X$ is convex,

- if $I \subset(0, \infty)$ and $X$ is harmonically convex and nondecreasing stochastic process then $X$ is convex,

- if $I \subset(0, \infty)$ and $X$ is convex and nondecreasing stochastic process then $X$ is harmonically convex.

The following result of the Hermite-Hadamard type inequalities holds. 
Theorem 3.1. Let $X: I \subset \mathbb{R} \backslash\{0\} \times \Omega \rightarrow \mathbb{R}$ be a harmonically convex stochastic process and $\mathrm{a}, \mathrm{b} \in \mathrm{I}^{\circ}$ with $a<b$. If $X \in L[a, b]$ then the following inequalities hold

$$
X\left(\frac{2 a b}{a+b},\right) \leq \frac{a b}{b-a} \int_{a}^{b} \frac{X(t, \cdot)}{t^{2}} d t \leq \frac{X(a, \cdot)+X(b, \cdot)}{2} .
$$

The above inequalities are sharp.

Proof. Since $X: I \times \Omega \rightarrow \mathbb{R}$ is a harmonically convex stochastic process, we have, for all $u, v \in$ $I$ (with $\lambda=1 \backslash 2$ in the inequality (1))

$$
X\left(\frac{2 u v}{u+v}, \cdot\right) \leq \frac{X(u, \cdot)+X(v, \cdot)}{2} .
$$

Choosing

$$
u=\frac{a b}{\lambda a+(1-\lambda) b}, v=\frac{a b}{\lambda b+(1-\lambda) a}
$$

we get

$$
X\left(\frac{2 u v}{u+v}, \cdot\right) \leq \frac{1}{2}\left\{X\left(\frac{a b}{\lambda a+(1-\lambda) b}, \cdot\right)+X\left(\frac{a b}{\lambda b+(1-\lambda) a}, \cdot\right)\right\} .
$$

Further, integrating for $\lambda \in[0,1]$ we have

$$
X\left(\frac{2 u v}{u+v}, \cdot\right) \leq \frac{1}{2}\left[\int_{0}^{1} X\left(\frac{a b}{\lambda a+(1-\lambda) b}, \cdot\right) d \lambda+\int_{0}^{1} X\left(\frac{a b}{\lambda b+(1-\lambda) a}, \cdot\right) d \lambda\right] .
$$

Since each of the integrals is equal to

$$
\frac{a b}{b-a} \int_{a}^{b} \frac{X(t, \cdot)}{t^{2}} d t
$$

we obtain the left-hand side of the inequality (2) from (3). The proof of the second inequality follows by using (1) with $u=a$ and $v=b$ and intgrating with respect to $\lambda$ over $[0,1]$. Now, consider the stochastic process $X:(0, \infty) \times \Omega \rightarrow \mathbb{R}, X(u, \cdot)=1$. Thus

$$
1=X\left(\frac{u v}{\lambda u+(1-\lambda) v}, \cdot\right)=\lambda X(v, \cdot)+(1-\lambda) X(u, \cdot)=1
$$

for all $u, v \in(0, \infty)$ and $\lambda \in[0,1]$. Therefore $X$ is harmonically convex on $(0, \infty)$. We also have

$$
X\left(\frac{2 a b}{a+b}, \cdot\right)=1, \frac{a b}{b-a} \int_{a}^{b} \frac{X(t, \cdot)}{t^{2}} d t=1, \frac{X(a, \cdot)+X(b, \cdot)}{2}=1
$$

which shows us the inequalities (2) are sharp.

Lemma 3.1. Let $X: I \subset \mathbb{R} \backslash\{0\} \times \Omega \rightarrow \mathbb{R}$ be a differentiable stochastic process on on $I^{\circ}$ and $\mathrm{a}, \mathrm{b} \in \mathrm{I}^{\circ}$ with $a<b$. If $X^{\prime} \in L[a, b]$ then

$\frac{X(a, \cdot)+X(b, \cdot)}{2}-\frac{a b}{b-a} \int_{a}^{b} \frac{X(t, \cdot)}{t^{2}} d t=\frac{a b(b-a)}{2} \int_{0}^{1} \frac{1-2 \lambda}{[\lambda b+(1-\lambda) a]^{2}} X^{\prime}\left(\frac{a b}{\lambda b+(1-\lambda) a}, \cdot\right) d \lambda$.

Proof. Let

$A=\frac{a b(b-a)}{2} \int_{0}^{1} \frac{1-2 \lambda}{[\lambda b+(1-\lambda) a]^{2}} X^{\prime}\left(\frac{a b}{\lambda b+(1-\lambda) \mathrm{a}}, \cdot\right) d \lambda$.

By integrating by part, we have

$A=\left.\frac{1-2 \lambda}{2} X\left(\frac{a b}{\lambda b+(1-\lambda) a}, \cdot\right)\right|_{0} ^{1}-\int_{0}^{1} X\left(\frac{a b}{\lambda b+(1-\lambda) a}, \cdot\right) d \lambda$

Setting $t=\frac{a b}{\lambda b+(1-\lambda) a}, d t=\frac{-a b(b-a)}{(\lambda b+(1-\lambda) a)^{2}} d \lambda=\frac{-t^{2}(b-a)}{a b} d \lambda$ we obtain

$A=\frac{X(a, \cdot)+X(b, \cdot)}{2}-\frac{a b}{b-a} \int_{a}^{b} \frac{X(t, \cdot)}{t^{2}} d t$ 
which gives the desired representation (4).

Theorem 3.2. Let $X: I \subset(0, \infty) \times \Omega \rightarrow \mathbb{R}$ be a differentiable stochastic process on $I^{o}$ and $a, b \in I^{o}$ with $\mathrm{a}<\mathrm{b}$, and $X^{\prime} \in L[a, b]$. If $\left|X^{\prime}\right|^{q}$ is harmonically convex on $[a, b]$ for $q \geq 1$, then

$$
\left|\frac{X(a, \cdot)+X(b, \cdot)}{2}-\frac{a b}{b-a} \int_{a}^{b} \frac{X(t, \cdot)}{t^{2}} d t\right| \leq \frac{a b(b-a)}{2} \lambda_{1}{ }^{1-\frac{1}{q}}\left[\lambda_{2}\left|X^{\prime}(a, \cdot)\right|^{q}+\lambda_{3}\left|X^{\prime}(b, \cdot)\right|^{q}\right]^{\frac{1}{q}}
$$

where

$$
\begin{aligned}
& \lambda_{1}=\frac{1}{a b}-\frac{2}{(b-a)^{2}} \ln \left(\frac{(a+b)^{2}}{4 a b}\right), \\
& \lambda_{2}=\frac{-1}{b(b-a)}+\frac{3 a+b}{(b-a)^{3}} \ln \left(\frac{(a+b)^{2}}{4 a b}\right), \\
& \lambda_{3}=\frac{1}{a(b-a)}-\frac{3 b+a}{(b-a)^{3}} \ln \left(\frac{(a+b)^{2}}{4 a b}\right)=\lambda_{1}-\lambda_{2} .
\end{aligned}
$$

Proof. From Lemma 3.1 and using Hölder inequality, we have

$$
\begin{aligned}
& \left|\frac{X(a, \cdot)+X(b, \cdot)}{2}-\frac{a b}{b-a} \int_{a}^{b} \frac{X(t, \cdot)}{t^{2}} \mathrm{~d} t\right| \\
& \leq \frac{a b(b-a)}{2} \int_{0}^{1}\left|\frac{1-2 \lambda}{[\lambda b+(1-\lambda) a]^{2}}\right|\left|X^{\prime}\left(\frac{a b}{\lambda b+(1-\lambda) a}, \cdot\right)\right| d \lambda \\
& \leq \frac{a b(b-a)}{2}\left(\int_{0}^{1} \frac{1-2 \lambda}{[\lambda b+(1-\lambda) a]^{2}} d \lambda\right)^{1-\frac{1}{q}} \times\left(\left|\frac{1-2 \lambda}{[\lambda b+(1-\lambda) a]^{2}}\right|\left|X^{\prime}\left(\frac{a b}{\lambda b+(1-\lambda) a}, \cdot\right)\right| d \lambda\right)^{\frac{1}{q}} .
\end{aligned}
$$

Hence, by harmonically convexity of If $\left|X^{\prime}\right|^{q}$ on $[a, b]$, we have

$$
\begin{aligned}
& \left|\frac{X(a, \cdot)+X(b, \cdot)}{2}-\frac{a b}{b-a} \int_{a}^{b} \frac{X(t, \cdot)}{t^{2}} d t\right| \\
& \leq \frac{a b(b-a)}{2}\left(\int_{0}^{1} \frac{|1-\lambda|}{[\lambda b+(1-\lambda) a]^{2}} d \lambda\right)^{1-\frac{1}{q}} \times\left(\int_{0}^{1} \frac{|1-2 \lambda|\left[\lambda\left|X^{\prime}(a, \cdot)\right|^{q}+(1-\lambda)\left|X^{\prime}(b, \cdot)\right|^{q}\right]}{[\lambda b+(1-\lambda) a]^{2}} d \lambda\right)^{\frac{1}{q}} \\
& \leq \frac{a b(b-a)}{2} \lambda_{1}{ }^{1-\frac{1}{q}}\left[\lambda_{2}\left|X^{\prime}(a, \cdot)\right|^{q}+\lambda_{3}\left|X^{\prime}(b, \cdot)\right|^{q}\right]^{\frac{1}{q}} .
\end{aligned}
$$

It is easily check that

$$
\begin{aligned}
& \int_{0}^{1} \frac{|1-2 \lambda|}{[\lambda b+(1-\lambda) a]^{2}} d \lambda=\frac{1}{a b}-\frac{2}{(b-a)^{2}} \ln \left(\frac{(a+b)^{2}}{4 a b}\right)=\lambda_{1}, \\
& \int_{0}^{1} \frac{|1-2 \lambda| \lambda}{[\lambda b+(1-\lambda) a]^{2}} d \lambda=\frac{-1}{b(b-a)}+\frac{3 a+b}{(b-a)^{3}} \ln \left(\frac{(a+b)^{2}}{4 a b}\right) \lambda_{2}, \\
& \int_{0}^{1} \frac{|1-2 \lambda|(1-\lambda)}{[\lambda b+(1-\lambda) a]^{2}} d \lambda=\frac{1}{a(b-a)}-\frac{3 b+a}{(b-a)^{3}} \ln \left(\frac{(a+b)^{2}}{4 a b}\right)=\lambda_{3}=\lambda_{1}-\lambda_{2} .
\end{aligned}
$$

Theorem 3.3. Let $X: I \subset(0, \infty) \times \Omega \rightarrow \mathbb{R}$ be a differentiable stochastic process on $\mathrm{I}^{\circ}$ and $a, b \in I^{o}$ with $a<b$ and $X^{\prime} \in L[a, b]$. If $\left|X^{\prime}\right|^{q}$ is harmonically convex on $[a, b]$ for $q>1, \frac{1}{p}+\frac{1}{q}=$ 1 , then

$$
\left|\frac{X(a, \cdot)+X(b, \cdot)}{2}-\frac{a b}{b-a} \int_{a}^{b} \frac{X(t, \cdot)}{t^{2}} d t\right| \leq \frac{a b(b-a)}{2}\left(\frac{1}{p+1}\right)^{\frac{1}{p}}\left[\mu_{1}\left|X^{\prime}(a, \cdot)\right|^{q}+\mu_{2}\left|X^{\prime}(b, \cdot)\right|^{q}\right]^{\frac{1}{q}}
$$

where

$$
\begin{aligned}
& \mu_{1}=\frac{a^{2-2 q}+b^{1-2 q}[(b-a)(1-2 q)-a]}{2(b-a)^{2}(1-q)(1-2 q)}, \\
& \mu_{2}=\frac{b^{2-2 q}-a^{1-2 q}[(b-a)(1-2 q)+b]}{2(b-a)^{2}(1-q)(1-2 q)},
\end{aligned}
$$


Proof. From Lemma 3.1 and using Hölder inequality and the harmonically convexity of $\left|X^{\prime}\right|^{q}$ on $[a, b]$, we have

$$
\begin{aligned}
& \left|\frac{X(a, \cdot)+X(b, \cdot)}{2}-\frac{a b}{b-a} \int_{a}^{b} \frac{X(t, \cdot)}{t^{2}} d t\right| \\
& \leq \frac{a b(b-a)}{2}\left(\int_{0}^{1}|1-2 \lambda|^{p} d \lambda\right)^{\frac{1}{p}} x\left(\frac{1}{[\lambda b+(1-\lambda) a]^{2 q}}\left|X^{\prime}\left(\frac{a b}{\lambda b+(1-\lambda) a}, \cdot\right)\right|^{q} d \lambda\right)^{\frac{1}{q}} \\
& \leq \frac{a b(b-a)}{2}\left(\frac{1}{p+1}\right)^{\frac{1}{p}} x\left(\int_{0}^{1} \frac{\lambda\left|X^{\prime}(a, \cdot)\right|^{q}+(1-\lambda)\left|X^{\prime}(b ; \cdot)\right|^{q}}{[\lambda b+(1-\lambda) a]^{2 q}} d \lambda\right)^{\frac{1}{q}} \\
& =\frac{a \mathrm{~b}(b-a)}{2}\left(\frac{1}{p+1}\right)^{\frac{1}{p}}\left[\mu_{1}\left|X^{\prime}(a, \cdot)\right|^{q}+\mu_{2}\left|X^{\prime}(b ; \cdot)\right|^{q}\right]^{\frac{1}{q}}
\end{aligned}
$$

where an easy calculation gives

$$
\begin{aligned}
& \int_{0}^{1} \frac{\lambda}{[\lambda b+(1-\lambda) a]^{2 q}} d \lambda=\frac{a^{2-2 q}+b^{1-2 q}[(b-a)(1-2 q)-a]}{2(b-a)^{2}(1-q)(1-2 q)}=\mu_{1}, \\
& \int_{0}^{1} \frac{1-\lambda}{[\lambda b+(1-\lambda) a]^{2 q}} d \lambda=\frac{b^{2-2 q}-a^{1-2 q}[(b-a)(1-2 q)+b]}{2(b-a)^{2}(1-q)(1-2 q)}=\mu_{2} .
\end{aligned}
$$

Substituting two equations into the above inequality, the result in Theorem is obtained, which completes the proof.

\section{Conclusion}

In this paper, we propose an important extension of convexity for stochastic processes which is called harmonically convex stochastic processes. These concepts are particularly interesting from optimization view point, since it provides a broader setting to study the optimization and mathematical programming problems. We also obtain the following Hermite-Hadamard type inequalities for harmonically convex stochastic processes under some suitable conditions:

$$
X\left(\frac{2 a b}{a+b},\right) \leq \frac{a b}{b-a} \int_{a}^{b} \frac{X(t, \cdot)}{t^{2}} d t \leq \frac{X(a, \cdot)+X(b ; \cdot)}{2} .
$$

In probabilistic words, it can be interpreted briefly as follows:

$$
X(E T, .) \leq_{s t} E X(T, .) \leq_{s t} E X\left(T^{*}, .\right), T \in \mathcal{C}_{s t} .
$$

Therefore, using Hermite-Hadamard type inequalities for harmonically convex stochastic processes are obtained some inequalities under some suitable conditions as follows:

$$
\begin{aligned}
& \left|\frac{X(a, \cdot)+X(b, \cdot)}{2}-\frac{a b}{b-a} \int_{a}^{b} \frac{X(t, \cdot)}{t^{2}} d t\right| \leq \frac{a b(b-a)}{2}\left(\frac{1}{p+1}\right)^{\frac{1}{p}}\left[\mu_{1}\left|X^{\prime}(a, \cdot)\right|^{q}+\mu_{2}\left|X^{\prime}(b, \cdot)\right|^{q}\right]^{\frac{1}{q}}, \\
& \left|\frac{X(a, \cdot)+X(b ; \cdot)}{2}-\frac{a b}{b-a} \int_{a}^{b} \frac{X(t, \cdot)}{t^{2}} d t\right| \leq \frac{a b(b-a)}{2}\left(\frac{1}{p+1}\right)^{\frac{1}{p}}\left[\mu_{1}\left|X^{\prime}(a, \cdot)\right|^{q}+\mu_{2}\left|X^{\prime}(b, \cdot)\right|^{q}\right]^{\frac{1}{q}} .
\end{aligned}
$$

Namely, these results can be interpreted probabilistically as follows:

$\left|E X\left(T^{*},.\right)-E X(T,).\right| \leq_{s t} \frac{a b(b-a)}{2} \lambda_{1}{ }^{1-\frac{1}{q}}\left[\lambda_{2}\left|X^{\prime}(a, \cdot)\right|^{q}+\lambda_{3}\left|X^{\prime}(b, \cdot)\right|^{q}\right]^{\frac{1}{q}}$,

$\left|E X\left(T^{*},.\right)-E X(T,).\right| \leq_{s t} \frac{a b(b-a)}{2}\left(\frac{1}{p+1}\right)^{\frac{1}{p}}\left[\mu_{1}\left|X^{\prime}(a, \cdot)\right|^{q}+\mu_{2}\left|X^{\prime}(b, \cdot)\right|^{q}\right]^{\frac{1}{q}}$. where $\quad \mathrm{E}$ denotes mathematical expectation, $X$ (respextively, $X^{*}$ ) is a stochastic process having the harmonically distribution on the interval $[a, b]$ (respectively, on the set $\{a, b\}$ ), and $\mathcal{C}_{s t}$ is the set of all real convex stochastic processes on $[a, b] ; \leq_{s t}$ stands for the so called convex order of stochastic processes.

These above inequalities are necessary to compare the maximum and minimum values of of a stochastic process with the expected value of its which has a probability density function, is particularly harmonic convex. 
As special cases, one can obtain several new and correct versions of the previously known results for various classes of these stochastic processes. Applying some type of inequalities for stochastic processes is another promising direction for future research.

\section{References}

Beckenbach, E., and Bellman, R. (1961). Inequalities. Springer, Berlin.

De la Cal, J. and Carcamo, J. (2006). Multidimensional Hermite-Hadamard Inequalities and The Convex Order. J. Math. Anal. Appl. 324, 248-261.

Dragomir, S.S. and Pearce, C.E.M. (2000). Selected Topics on Hermite-Hadamard Inequality and Applications. Victoria University, Melbourne.

Galler, R.G. (2013). Stochastic Processes Theory for Applications. Cambridge University Press, New York.

Josip E., Pecaric J.E, Proschan, F. and Thong, Y.L. (1992). Convex Functions, Parital Orderings, and Statistical Applications. Mathematics in Science and Engineering Series, Academic Press.

İşcan, i. (2014). Hermite-Hadamard Inequalities for Harmonically Convex Functions. Hacet. J. Math. Stat. 43 (6), 935-942.

Knill, O. (2009). Probability and Stochastic Processes with Applications. Overseas Press, New Delhi.

Kotrys, D. (2012) Hermite-Hadamard Inequality for Convex Stochastic Processes. Aequat. Math. 83, 143-151.

Mitrinovic, D.S. (1970). Analytic Inequalities. Springer-Verlang, Berlin.

Nikodem, K. (1980). On Convex Stochastic Processes. Aequat. Math. 20, 184-197.

Shaked, M. and Shanthikumar, J.G. (1988) Stochastic Convexity and Its Applications. Advances in Applied Probability. 20, 427-446.

Simon, B. (2011). Convexity. An Analytic Viewpoint, Cambridge Tracts in Mathematics. Cambridge University Press, New York.

Skowronski, A. (1992). On Some Properties of J-convex Stochastic Processes. Aequat. Math. 44, 249-258.

Skowronski, A. (1995.). On Wright-Convex Stochastic Processes. Annales Math. 9, 29-32.

Suhov, Y. and Kelbert, M. (2005). Probability and Statistics by Example. Cambridge University Press, New York. 
